\title{
Linear precoding design for massive MIMO based on the minimum mean square error algorithm
}

\author{
Zhou Ge 1* $^{*}$ and Wu Haiyan ${ }^{2}$
}

\begin{abstract}
Compared with the traditional multiple-input multiple-output (MIMO) systems, the large number of the transmit antennas of massive MIMO makes it more dependent on the limited feedback in practical systems. In this paper, we study the problem of precoding design for a massive MIMO system with limited feedback via minimizing mean square error (MSE). The feedback from mobile users to the base station (BS) is firstly considered; the BS can obtain the quantized information regarding the direction of the channels. Then, the precoding is designed by considering the effect of both noise term and quantization error under transmit power constraint. Simulation results show that the proposed scheme is robust to the channel uncertainties caused by quantization errors.
\end{abstract}

Keywords: Massive MIMO, Precoding, Minimum mean square error (MMSE), Limited feedback

\section{Introduction}

Multiple-input multiple-output (MIMO) techniques have gained considerable attention in modern wireless communications since it can significantly improve the capacity and reliability of wireless systems [1]. The essence of downlink multiuser MIMO is precoding, which means that the antenna arrays are used to direct each data signal spatially towards its intended receiver. Unfortunately, the precoding design in multiuser MIMO requires very accurate instantaneous channel state information (CSI) [2] which can be cumbersome to achieve in practice. To further achieve more dramatic gains as well as to simplify the required signal processing, massive MIMO techniques have been proposed in $[3,4]$ by installing a large number of antennas at base stations (BS), possibly in the order of tens or hundreds, which promises significant performance gains in terms of spectral efficiency and energy efficiency compared with conventional MIMO and is becoming a cornerstone of future $5 \mathrm{G}$ systems $[5,6]$.

From a practical point of view, realizing massive MIMO systems has to deal with several challenges, one

\footnotetext{
*Correspondence: zhougels@sina.com

${ }^{1}$ Department of Information Engineering, Chongqing Youth Vocational and

Technical College, Chongqing, People's Republic of China

Full list of author information is available at the end of the article
}

of which is the low-complexity and near-optimal precoding scheme [7, 8]. Generally, precoding approaches can be classified into nonlinear precoding and linear precoding. The optimal precoding is the nonlinear dirty paper precoding (DPC) [9], which can effectively eliminate the interference between different users and achieve optimal performance. However, nonlinear precoding schemes usually suffer from high complexity which makes them unpractical due to the hundreds of antennas in massive MIMO systems. Since the asymptotic orthogonality of massive MIMO channel matrix, simple linear precoding (e.g., zero-forcing (ZF) precoding) can be used to achieve capacity-approaching performance. Nevertheless, ZF precoding requires matrix inversion of very large size, which exhibits prohibitively high complexity. To reduce the complexity of matrix inversion of large size, a Neumann-based precoding is proposed in [10] to reduce the computational complexity in an iterative method, but the required complexity is still unaffordable. Recently, a low peak-to-average power ratio (PAPR) precoding based on the approximate message passing (AMP) algorithm [11] and a successive over-relaxation (SOR)-based precoding [12] are respectively proposed to minimize multiuser interference (MUI) in massive multiuser MIMO systems. The aforementioned works are based on the assumption of a perfect CSI at the BS, 
which is somewhat too optimistic for practical applications. As a result, it is essential to investigate the robust precoding design in massive MIMO systems.

Inspired by the abovementioned works, in this paper, we study the precoding design for a single-cell downlink massive MIMO system with limited feedback, while guaranteeing transmit power constraint. Each user terminal (UT) feeds back the quantized side information to BS to assist its transmission. We propose a linear precoder design scheme via the minimum mean square error (MMSE) criteria with respect to the CSI imperfection. The proposed scheme is an improved approach, which is robust to the channel uncertainties caused by quantization errors and the lack of channel quality information (CQI).

The rest of this paper is organized as follows. In Section 1, the system mode of massive MIMO is introduced and the problem is formulated. In Section 2, a linear precoder based on MMSE criteria is designed by considering the impact of the noise term and CSI quantization error. Numerical results are presented in Section 3. Finally, concluding remarks are made in Section 4.

Notations: Throughout this paper, boldface lowercase and uppercase letters denote vectors and matrices, respectively. The transpose, conjugate transpose, trace, and Frobenius norm of a matrix $\mathbf{A}$ are denoted as $\mathbf{A}^{\mathrm{T}}$, $\mathbf{A}^{\mathrm{H}}, \operatorname{tr}(\mathbf{A}),\|\mathbf{A}\|_{F}$ respectively. $I_{M \times M}$ denotes a $M \times M$ identity matrix. $E[\cdot]$ denotes the expectation operator. $\operatorname{diag}($.$) stands for a diagonal matrix with the given ele-$ ments on the diagonal. $\operatorname{Re}($.$) represents the real part of$ the input.

\section{System model}

We consider a single-cell downlink multiuser massive MIMO system, as depicted in Fig. 1. For massive MIMO downlink transmissions, a large number of antennas $N_{T}$ that are equipped at the BS is serving $K$ UTs with each UT being equipped with $n_{r}$ antennas and the total

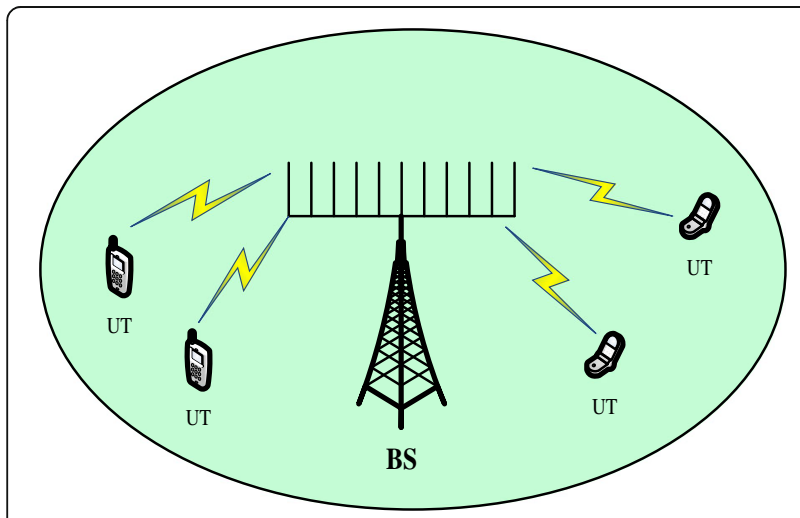

Fig. 1 System model receive antennas of UTs is $N_{R}=K n_{r}$. Here, $\mathbf{H} \in C^{N_{T} \times N_{R}}$ $=\left[\mathbf{H}_{1}, \mathbf{H}_{2}, \ldots, \mathbf{H}_{K}\right]$ denotes the fast fading channel matrix from BS to the UTs, where each element is a zero mean unit variance independent and identical distributed (i.i.d) complex Gaussian. Let $\mathbf{D}=\mathbf{H D}_{\mu^{1 / 2}}$, thus $\mathbf{D}_{\mu^{1 / 2}}=$ diag $\left\{\sqrt{\mu_{1}}, \sqrt{\mu_{2}}, \ldots, \sqrt{\mu_{K}}\right\}$ denotes slow fading diagonal matrix.

Then, the total received signal $\mathbf{y}=\left[\mathbf{y}_{1}^{\mathrm{T}}, \mathbf{y}_{2}^{\mathrm{T}}, \ldots, \mathbf{y}_{K}^{\mathrm{T}}\right]^{\mathrm{T}}$ at all UTs is given by

$$
\mathbf{y}=\mathbf{D}^{\mathrm{H}} \mathbf{W} \mathbf{x}+\mathbf{n},
$$

where $\mathbf{x}$ is the signals transmitted by the BS and $\mathbf{n}$ is an additive white Gaussian noise with zero mean and variance $\sigma^{2} . \mathbf{W}=\left[\mathbf{W}_{1}, \mathbf{W}_{2}, \cdots, \mathbf{W}_{K}\right]$ is the transmitting precoding matrix. Denoting $P_{T}$ as the power constraint at $\mathrm{BS}$, the total transmit power at BS is limited by $\operatorname{tr}\left(\mathbf{W} \mathbf{W}^{\mathrm{H}}\right) \leq P_{T}$.

We assume that each UT can perfectly estimate the downlink CSI and send it back to ST using local feedback. All of the feedback channels are assumed to be noiseless and delay free. To facilitate analysis, the channel is decomposed into the channel direction information (CDI) and CQI [11]. The kth UT estimates the CSI of channel $\mathbf{D}_{k}$ perfectly and quantizes the CDI $\tilde{\mathbf{D}}_{k}=\mathbf{D}_{k} /\left\|\mathbf{D}_{k}\right\|$ to a unit norm vector $\hat{\mathbf{D}}_{k}$.

The acquisition of $\mathbf{D}_{k}$ at $\mathrm{BS}$ can be accomplished via channel feedback. The quantized CDI $\hat{\mathbf{D}}_{k}$ is chosen from a predefined codebook $C$ that consists of $2^{B}$ codeword matrices $\left\{C_{1}, C_{2}, \cdots, C_{2^{B}}\right\}$, where $B$ is the bit size of the codeword vector. Each UT quantizes its channel to the quantization vector that is closest to its channel vector, where closeness is measured in terms of the angle between two vectors or, equivalently, the inner product $[12,13]$. Thus, the quantization of $k$ th UT is chosen according to the minimum angle criterion as

$$
\begin{aligned}
& \hat{\mathbf{D}}_{k}=\arg \max _{C_{i} \in C}\left|\tilde{\mathbf{D}}_{k}^{\mathrm{H}} C_{i}\right| \\
& =\arg \min _{C_{i} \in C} \sin ^{2}\left(\angle\left(\tilde{\mathbf{D}}_{k}, C_{i}\right)\right),
\end{aligned}
$$

and each UT feeds back the $B$ bits codeword indices to the BS. Due to simplicity and analytical tractability, we employ random vector quantization (RVQ) for the codebook design where $\left\{C_{i}\right\}_{i=1}^{2^{B}}$ are chosen independently and isotropically on the $N_{T^{-}}$-dimensional unit sphere [14]. Throughout the paper, we assume that this CQI is known perfectly to the BS, i.e., it is not quantized; thus, no CQI is fed back to the BS.

\section{Linear precoder design}

In this section, we introduce a linear precoder design scheme that considers the effect of both noise term and quantization error. 


\subsection{Channel model}

In the following, according to [15], the subspace of the true channel matrix can be decomposed as the weighted sum of the quantized channel and an independent and isotropic quantization error term.

$$
\tilde{\mathbf{D}}_{k}=\hat{\mathbf{D}}_{k} \mathbf{X}_{k} \mathbf{Y}_{k}+\mathbf{S}_{k} \mathbf{Z}_{k} n_{r}
$$

where $\mathbf{D}_{k}$ is an orthonormal basis for the subspace spanned by the columns of $\mathbf{D}_{k} . \mathbf{X}_{k} \in C^{n_{r} \times n_{r}}$ is a unitary matrix; $\mathbf{Y}_{k} \in C^{n_{r} \times n_{r}}$ is upper triangular with positive diagonal elements and satisfies $\mathbf{Y}_{k}^{\mathrm{H}} \mathbf{Y}_{k}=\mathbf{I}_{n_{r}}-\mathbf{Z}_{k}^{\mathrm{H}} \mathbf{Z}_{k} . \mathbf{Z}_{k} \in C^{n_{r} \times n_{r}}$ is upper triangular with positive diagonal elements and represents the quantization error, satisfying $\operatorname{tr}\left(\mathbf{Z}_{k}^{\mathrm{H}} \mathbf{Z}_{k}\right)$ $=\sin ^{2}\left(\angle\left(\tilde{\mathbf{D}}_{k}, \hat{\mathbf{D}}_{k}\right)\right) . \mathbf{S}_{k}$ is an orthonormal basis for an isotropically distributed $n_{r}$-dimensional plane in the left null space of $\hat{\mathbf{D}}_{k}$.

Therefore, the broadcast channel $\mathbf{D}_{k}$ can be decomposed as

$$
\mathbf{D}=\left[\hat{\mathbf{D}}\left(\mathbf{I}_{N_{R}}-\mathbf{Z}^{\mathrm{H}} \mathbf{Z}\right)^{1 / 2}+\mathbf{S Z}\right] \mathbf{R}=\hat{\mathbf{D}} \mathbf{A}+\mathbf{S B},
$$

where $\mathbf{R}=\operatorname{diag}\left(\sqrt{\Lambda_{1}}, \sqrt{\Lambda_{2}}, \ldots, \sqrt{\Lambda_{K}}\right)$ and $\mathbf{Z}=\operatorname{diag}\left(\mathbf{Z}_{1}\right.$, $\left.\mathbf{Z}_{2}, \ldots, \mathbf{Z}_{K}\right)$ and $\Lambda_{k}$ is a diagonal matrix that consists of $n_{r}$ non-zero eigenvalues of $\mathbf{D}_{k} \mathbf{D}_{k}^{\mathrm{H}} \cdot \mathbf{A}=\left(\mathbf{I}_{N_{R}}-\mathbf{Z}^{\mathrm{H}} \mathbf{Z}\right)^{1 / 2} \mathbf{R}$ and $\mathbf{B}=\mathbf{Z R}$.

The achievable rate of the $k$ th UT can be expressed as

$$
\begin{aligned}
R & =E\left[\log _{2} \frac{\left|\mathbf{I}_{N_{R}}+\sum_{i=1}^{K} \mathbf{D}_{k}^{\mathrm{H}} \mathbf{W}_{i} \mathbf{W}_{i}^{\mathrm{H}} \mathbf{D}_{k}\right|}{\left|\mathbf{I}_{N_{R}}+\sum_{i=1, i \neq k}^{K} \mathbf{D}_{k}^{\mathrm{H}} \mathbf{W}_{i} \mathbf{W}_{i}^{\mathrm{H}} \mathbf{D}_{k}\right|}\right] \\
& =E\left[\log _{2}\left|\mathbf{I}_{N_{R}}+\sum_{i=1}^{K} \mathbf{D}_{k}^{\mathrm{H}} \mathbf{W}_{i} \mathbf{W}_{i}^{\mathrm{H}} \mathbf{D}_{k}\right|\right] \\
& -E\left[\log _{2}\left|\mathbf{I}_{N_{R}}+\sum_{i=1, i \neq k}^{K} \mathbf{D}_{k}^{\mathrm{H}} \mathbf{W}_{i} \mathbf{W}_{i}^{\mathrm{H}} \mathbf{D}_{k}\right|\right] .
\end{aligned}
$$

\subsection{Linear precoder design}

The precoders can be designed to alleviate multiuser interference, maximize the received desired signal power, or minimize the MSE of the received signals. We in the following will take the MMSE precoder as an example. Then, MMSE cost function can be defined as [15]

$$
\min _{\beta, \mathbf{W}} E\left(\left\|\mathbf{x}-\beta^{-1} \mathbf{y}\right\|_{F}^{2}\right) \text { s.t. } E\left(\|\mathbf{W} \mathbf{x}\|_{F}^{2}\right) \leq P_{T}
$$

Substituting (4) into (6), the conditional expectation becomes

$$
\begin{aligned}
\mathrm{E}\left(\left\|\mathbf{x}-\beta^{-1} \mathbf{y}\right\|_{F}^{2}\right)= & E_{\mathbf{A}, \mathbf{B}, \mathbf{x}}\left(\left\|\mathbf{x}-\beta^{-1} \mathbf{A}^{\mathrm{H}} \hat{\mathbf{D}}^{\mathrm{H}} \mathbf{W} \mathbf{x}-\beta^{-1} \mathbf{B}^{\mathrm{H}} \mathbf{S}^{\mathrm{H}} \mathbf{W} \mathbf{x}-\beta^{-1} \mathbf{n}\right\|_{F}^{2}\right) \\
= & \beta^{-2} E_{\mathbf{A}}\left[\operatorname{tr}\left(\mathbf{W}^{\mathrm{H}} \hat{\mathbf{D}} \mathbf{A} \mathbf{A}^{\mathrm{H}} \hat{\mathbf{D}}^{\mathrm{H}} \mathbf{W}\right)\right] \\
& +\beta^{-2} E_{\mathbf{B}, \mathbf{S}}\left[\operatorname{tr}\left(\mathbf{W}^{\mathrm{H}} \mathbf{S B B B} \mathbf{B}^{\mathrm{H}} \mathbf{S}^{\mathrm{H}} \mathbf{W}\right)\right] \\
& +\left(\beta^{-2}+1\right)\left\|\mathbf{I}_{N_{R}}\right\|_{F}^{2}-\beta^{-1} E_{\mathbf{A}}\left[\operatorname{tr}\left(\mathbf{W}^{\mathrm{H}} \hat{\mathbf{D}} \mathbf{A}\right)\right] \\
& -\beta^{-1} E_{\mathbf{A}}\left[\operatorname{tr}\left(\mathbf{A}^{\mathrm{H}} \hat{\mathbf{D}}^{\mathrm{H}} \mathbf{W}\right)\right]
\end{aligned}
$$

The first term in (7) can be further calculated as

$$
\begin{aligned}
& \beta^{-2} E_{\mathbf{A}}\left[\operatorname{tr}\left(\mathbf{W}^{\mathrm{H}} \hat{\mathbf{D}} \mathbf{A} \mathbf{A}^{\mathrm{H}} \hat{\mathbf{D}}^{\mathrm{H}} \mathbf{W}\right)\right] \\
& =\beta^{-2} E_{\mathbf{R}, \mathbf{Z}}\left[\operatorname{tr}\left(\mathbf{R}\left(\mathbf{I}_{N_{R}}-\mathbf{Z} \mathbf{Z}^{\mathrm{H}}\right) \mathbf{W}^{\mathrm{H}} \hat{\mathbf{D}} \hat{\mathbf{D}}^{\mathrm{H}} \mathbf{W}\right)\right] \\
& =\beta^{-2}\left(N_{T}-\frac{N_{T} \Delta}{N_{R}}\right) \operatorname{tr}\left(\mathbf{W}^{\mathrm{H}} \hat{\mathbf{D}} \hat{\mathbf{D}}^{\mathrm{H}} \mathbf{W}\right),
\end{aligned}
$$

where $E\left[\mathbf{R R}^{\mathrm{H}}\right]=E\left[\operatorname{diag}\left\{\Lambda_{1}, \Lambda_{2}, \ldots, \Lambda_{K}\right\}\right]=N_{T} \mathbf{I}_{N_{R}}, \quad E$ $\left[\mathbf{Z Z}^{\mathrm{H}}\right]=\frac{\Delta}{N_{R}} \mathbf{I}_{N_{R}}$. Here, $\Delta$ denotes the quantization error that can be approximated as [16]

$$
\Delta=\frac{\Gamma\left(\frac{1}{T}\right)}{T} \Phi^{-\frac{1}{T}} 2^{-\frac{B}{T}}
$$

where $T=n_{r}\left(N_{T}-n_{r}\right)$ and $\Phi=\frac{1}{T !} \prod_{m=1}^{n_{r}} \frac{\left(N_{T}-m\right) !}{\left(n_{r}-m\right) !} \cdot \Gamma(\cdot)$ de-
notes gamma function. The second item in (7) can also be calculated as

$$
\begin{aligned}
& \beta^{-2} E_{\mathbf{B}, \mathbf{S}}\left[\operatorname{tr}\left(\mathbf{W}^{\mathrm{H}} \mathbf{S B B} B^{\mathrm{H}} \mathbf{S}^{\mathrm{H}} \mathbf{W}\right)\right] \\
& =\beta^{-2} P_{T}\left(\frac{N_{T} \Delta}{N_{T}-n_{r}}\right)-\beta^{-2}\left(\frac{N_{T} n_{r} \Delta}{N_{R}\left(N_{T}-n_{r}\right)}\right) .
\end{aligned}
$$

By substituting (8) and (10) into (7), the optimization problem can be rewritten by

$$
\begin{aligned}
E( & \left.\left\|\mathbf{x}-\beta^{-1} \mathbf{y}\right\|_{F}^{2}\right) \\
= & \beta^{-2}\left(N_{T}-\frac{N_{T}^{2} \Delta}{N_{R}\left(N_{T}-n_{r}\right)}\right) \operatorname{tr}\left(\mathbf{W}^{\mathrm{H}} \hat{\mathbf{D}} \hat{\mathbf{D}}^{\mathrm{H}} \mathbf{W}\right)+\frac{\beta^{-2} N_{T} \Delta}{N_{T}-n_{r}} \\
& +\left(\beta^{-2}+1\right) N_{R}-2 \alpha \beta^{-1} \operatorname{Re}\left[\operatorname{tr}\left(\mathbf{W}^{\mathrm{H}} \hat{\mathbf{D}}\right)\right], F(\mathbf{W}, \beta)
\end{aligned}
$$

where $\alpha \mathbf{I}_{N_{R}}=E[\mathbf{A}]=E\left[\left(\mathbf{I}_{N_{R}}-\mathbf{Z}^{\mathrm{H}} \mathbf{Z}\right)^{1 / 2} \mathbf{R}\right]$.

Therefore, the optimization problem in (6) is equivalent to

$$
\min _{\beta, \mathbf{W}} F(\mathbf{W}, \beta) \quad \text { s.t. } \operatorname{tr}\left(\mathbf{W}^{\mathrm{H}} \mathbf{W}\right) \leq P_{T} \quad .
$$

Obviously, problem (12) is a convex optimization problem. Hence, problem (12) can be solved with a closed-form solution by exploiting the KarushKuhn-Tucker (KKT) conditions [17]. Constructing the Lagrangian function, we have 


$$
L(\mathbf{W}, \beta, \lambda)=F(\mathbf{W}, \beta)+\lambda\left[\operatorname{tr}\left(\mathbf{W}^{\mathrm{H}} \mathbf{W}\right)-P_{T}\right] .
$$

The optimal solution to $\mathbf{W}$ can be calculated by taking the first-order derivative of (13) with respect to $\mathbf{W}$ and setting it to zero, i.e., $\frac{\partial L(\mathbf{W}, \beta, \lambda)}{\partial \mathbf{W}}=0$. We can easily get

$$
\mathbf{W}=\rho\left(\hat{\mathbf{D}} \hat{\mathbf{D}}^{\mathrm{H}}+\eta \mathbf{I}_{N_{R}}\right)^{-1} \hat{\mathbf{D}}
$$

where $\rho=\sqrt{\frac{P_{T}}{\rho\left(\hat{\mathbf{D}} \hat{\mathbf{D}}^{\mathrm{H}}+\eta \mathbf{I}_{N_{R}}\right)^{-1} \hat{\mathbf{D}}}}(\rho$ is normalized power factor) and $\eta=\frac{P_{T} N_{T} \Delta+N_{R}\left(N_{T}-n_{r}\right)}{P_{T}\left[\left(N_{T}-n_{r}\right)-N_{T}^{2} \Delta\right]}$.

Updating the Lagrange multiplier $\lambda$, we have

$$
\lambda(t+1)=\left[\lambda(t)+a_{1}\left(\operatorname{tr}\left(\mathbf{W}^{\mathrm{H}} \mathbf{W}\right)-P_{T}\right)\right]^{+},
$$

where $[X]^{+}=\max \{X, 0\} . a_{1}$ is the step size which is positive and $t$ is the step time.

(1) If $\Delta=0$, that is perfect CSI. Here, $\eta=\frac{N_{R}}{P_{T}}$. The precoding matrix can be rewritten as

$$
\mathrm{W}=\rho\left(\hat{\mathrm{D}} \hat{\mathrm{D}}^{\mathrm{H}}+\eta \mathrm{I}_{N_{R}}\right)^{-1} \hat{\mathrm{D}}
$$

(2) If $\Delta \neq 0$, that is imperfect CSI. Here, $n_{r}=1$ and $N_{T}$ $=N_{R}=K$ for simplicity, $\Delta=\frac{N_{T}-1}{N_{T}} 2^{-\frac{B}{N_{T}^{-1}}}$. The precoding matrix can be rewritten as

$$
\mathrm{W}=\rho\left[\frac{\hat{\mathrm{D}} \hat{\mathrm{D}}^{\mathrm{H}}+P_{T} 2^{-\frac{B}{N_{T}-1}}+N_{T}}{P_{T}\left(1-N_{T} 2^{-\frac{B}{N_{T}-1}}\right) \mathrm{I}_{N_{R}}}\right]^{-1} \hat{\mathrm{D}} .
$$

Here, we use the sub-gradient algorithm to solve the problem. Using a constant step length $t_{1}$ and $t_{2}$, the sub-gradient algorithm can converge to the optimal point of convex problems within a small range.

To summarize, the procedure of the sub-gradient algorithm based on updating (15) is shown in Table 1.

Since MMSE function in (12) is convex on a single precoder $\mathbf{W}$, updating $\mathbf{W}$ at each iteration monotonically reduces the MMSE in (12), which is lower bounded by zero. Algorithm 1 can converge to the optimal point of the problem (6) within a small

Table 1 The proposed sub-gradient algorithm

Initialization: $0<\lambda<1$

Repeat: At each iteration $t, t=0,1,2, \ldots$

- Precoding update: update precoding vector $\mathbf{W}$ using equation (17).

- Lagrangian multiplier update: update the Lagrangian multiplier $\lambda$ using equation (15).

Until: $\lambda$ converges. range. Although the precoder design depends on inaccurate CSI feedback, it may not always satisfy the transmit power constraint. However, we can assume a procedure that a feedforward link exists between UTs and BS. Each UT sends information from the precoder $\mathbf{W}$ to the BS via the feedforward link, then BS estimates the received power to satisfy the transmit power constraint.

\subsection{Analysis of computational complexity}

In this subsection, we analyze the computational complexity of the proposed precoder and compare it to the complexity of ZF precoder. We express the computational complexity in terms of the number of floating point operations (FLOPs). Following [18], the complexity of our proposed scheme can be calculated as

$$
\tau\left[(K+1)(2 K-1) N_{T}+\left(2 N_{T}-1\right) K\right],
$$

where $\tau$ is the number of transmit symbols per user, $K$ is the number of UTs, and $N_{T}$ is the transmit antennas at BS. As the simplest precoding scheme, the computational complexity of the ZF precoder can be calculated as $\tau N_{T}(2 K-1)$ FLOPs.

\section{Simulation results}

In this section, the performance of the proposed scheme is evaluated by a computer simulation. In our simulations, the elements of all the signaling channel matrices are assumed to be i.i.d. complex Gaussian variables with zero means and unit variance. We assume that the number of UTs is $K=30$, the total transmit power is $P_{T}=$ $20 \mathrm{~dB}$, and the background noise is $\sigma^{2}=1$.

For simplicity, we assume that the received number of antennas at each UT is $n_{r}=1$.

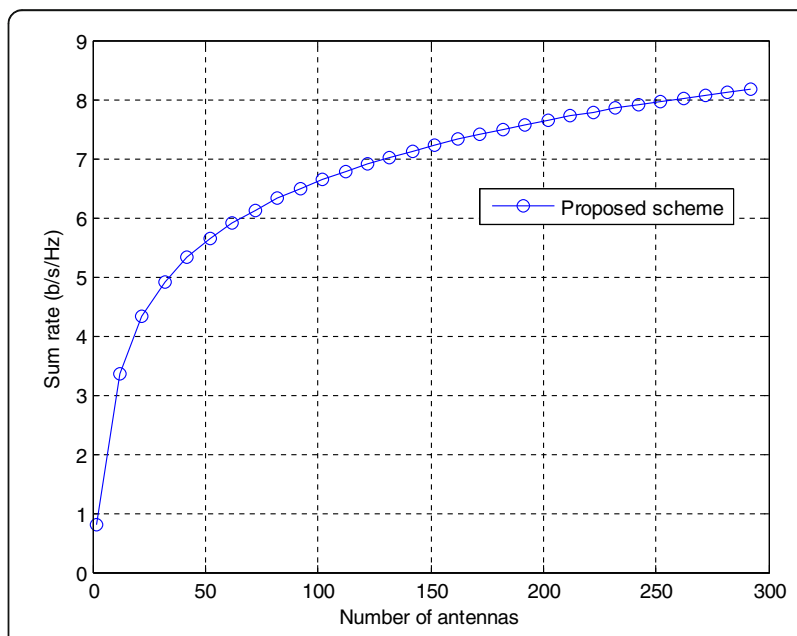

Fig. 2 Sum rate versus the number of transmit antennas $N_{T}$ 


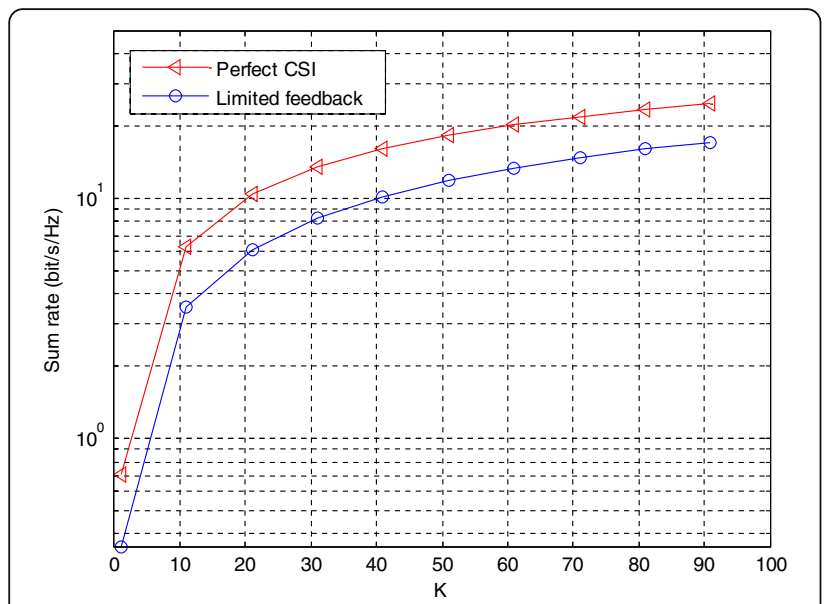

Fig. 3 Sum rate versus the number of UTs $K$

Figure 2 depicts the effect of the number of antennas on the sum rate of the proposed algorithm with $K=6$. As shown in Fig. 2, the sum rate increases with the increasing of the number of transmit antennas. It is observed that when the number of transmit antennas is more than 250 , the sum rate gradually trends to saturation.

Figure 3 shows the sum rate as a function of the number of UTs under perfect CSI and limited feedback. It is observed that the sum rate increases as the number of the number of UTs increases. Clearly, there is a narrow gap between the proposed limited feedback scheme and perfect CSI.

Figure 4 shows the sum rate performance of different schemes under increasing number of feedback bits $B$ under transmit antennas $N_{T}=160$ and $n_{r}=1$. We take the traditional $\mathrm{BD}-\mathrm{ZF}$ and conventional MMSE (C-MMSE) into comparison. It is clearly observed that our proposed scheme achieves a higher sum rate than other schemes, expect for perfect transmit CSI. We also find that the proposed scheme overcomes the sum rate degradation problem at high-SNR regions that the C-MMSE scheme has encountered.

\section{Conclusions}

In this paper, we investigated the problem of linear precoding design for massive MIMO system in a single cell based on MMSE criteria under transmit power constraint. The proposed scheme was robust to the uncertainties in the CSI as it taken into account the effect of quantization errors and noise term. Simulation results show the superiority of our proposed quantization scheme. In the future work, we plan to study the partial feedback of CSI for multicell massive MIMO systems.

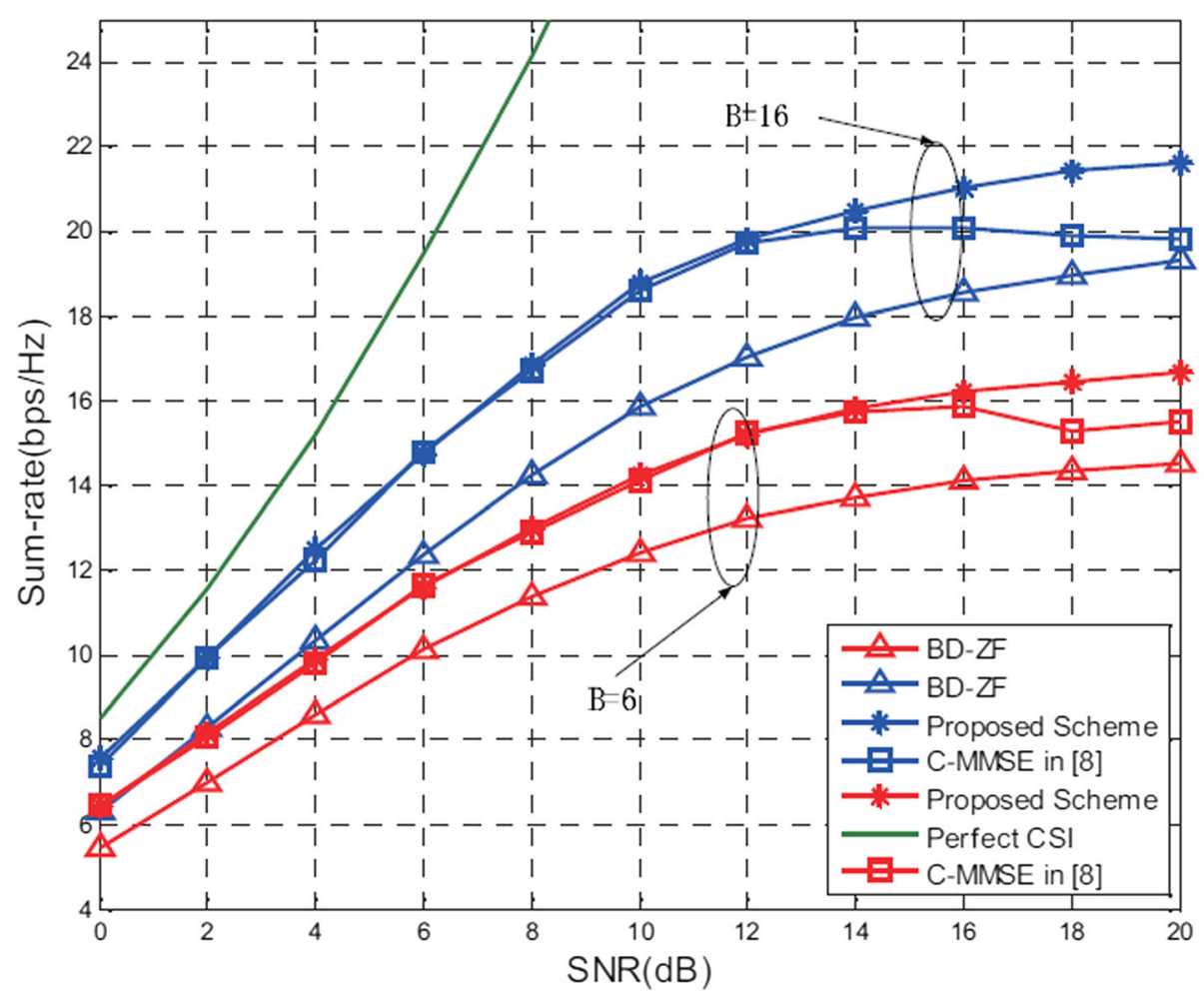

Fig. 4 Performance comparison of the proposed scheme with different schemes under $B=6$ bit and $B=16$ bit 


\section{Competing interests}

The authors declare that they have no competing interests.

\section{Author details}

'Department of Information Engineering, Chongqing Youth Vocational and Technical College, Chongqing, People's Republic of China. ${ }^{2}$ Department of Police Management Railway Police College, Zhengzhou, Henan 450003, People's Republic of China.

Received: 10 March 2016 Accepted: 29 November 2016

Published online: 25 January 2017

\section{References}

1. C Lim, T Yoo, B Clerckx, B Lee, B Shim, Recent trend of multiuser MIMO in LTE-Advanced. IEEE Commun. Mag. 51(3), 127-135 (2013)

2. D Gesbert, M Kountouris, RW Heath, C-B Chae, T Sälzer, Shifting the MIMO paradigm. IEEE Signal Process. Mag. 24(5), 36-46 (2007)

3. N Jindal, MIMO broadcast channels with finite-rate feedback. IEEE Trans. Inf. Theory 52(11), 5045-5060 (2006)

4. TL Marzetta, Noncooperative cellular wireless with unlimited numbers of base station antennas. IEEE Trans. Wirel. Commun. 9(11), 3590-3600 (2010)

5. L Lu, GY Li, AL Swindlehurst, A Ashikhmin, R Zhang, An overview of massive MIMO: benefits and challenges. IEEE J. Sel. Areas Commun. 8(5), 742-758 (2014)

6. F Boccardi, RW Heath Jr, A Lozano, TL Marzetta, P Popovski, Five disruptive technology directions for 5G. IEEE Commun. Mag. 52(2), 74-80 (2014)

7. MH Costa, Writing on dirty paper (corresp.). IEEE Trans. Inf. Theory 29(3), 439-441 (1983)

8. H Prabhu, J Rodrigues, O Edfors, F Rusek, Approximative Matrix Inverse Computations for Very-Large MIMO and Applications to Linear Pre-coding Systems (Proceedings of IEEE Wireless Communications and Networking Conference (WCNC), Shanghai, China, 2013), pp. 2710-2715. April 7-10

9. JC Chen, CJ Wang, KK Wong, et al., Low-complexity precoding design for massive multiuser MIMO systems using approximate message passing. IEEE Trans. Vehicular Technol. 65(7), 5707-5714 (2016)

10. T Xie, Q Han, H Xu, Z Qi, W Shen, A Low-Complexity Linear Precoding Scheme Based on SOR Method for Massive MIMO Systems (Proceedings of the 81st IEEE Vehicular Technology Conference (VTC-Spring), Glasgow, 2015), pp. 1 5. May 11-14

11. T Yoo, N Jindal, A Goldsmith, Multi-antenna downlink channels with limited feedback and user selection. IEEE J. Sel. Areas Commun. 25(7), 1478-1491 (2007)

12. LY Song, Z Han, ZS Zhang, BL Jiao, Non-cooperative feedback-rate control game for channel state information in wireless networks. IEEE J. Sel. Areas Commun. 30(1), 188-197 (2012)

13. J-H Noh, S-J Oh, Beamforming in a multi-user cognitive radio system with partial channel state information. IEEE Trans. Wirel. Commun. 12(2), 616-625 (2013)

14. N Ravindran, N Jindal, Multi-user diversity vs. accurate channel state information in MIMO downlink channels. IEEE Trans. Wirel. Commun. 11(9), 201-220 (2012)

15. K-J Lee, I Lee, MMSE based block diagonalization for cognitive radio MIMO broadcast channels. IEEE Trans. Wirel. Commun. 10(10), 3139-3144 (2011)

16. N Ravindran, N Jindal, Limited feedback-based block diagonalization for the MIMO broadcast channel. IEEE J. Sel. Areas Commun. 26(8), 1473-1482 (2008)

17. S Boyd, L Vandenberghe, Convex Optimization (Cambridge Univ. Press, Cambridge, U.K., 2004)

18. C Guthy, W Utschick, R Hunger, et al., Weighted sum rate maximization in the MIMO MAC with linear transceivers: algorithmic solutions Asilomar Conference on. IEEE Xplore. 2009, 1528-1532

\section{Submit your manuscript to a SpringerOpen ${ }^{\circ}$ journal and benefit from:}

- Convenient online submission

- Rigorous peer review

- Immediate publication on acceptance

- Open access: articles freely available online

- High visibility within the field

- Retaining the copyright to your article 